\title{
Long Distance Call
}

\author{
Keywords \\ Arduino, Augmented Space, Imaginary, Interactivity, Interface.
}

The paper covers three interactive installations carried out by the artist-authorresearcher in his artistic/poetic research project "Interurbano" (long distance call) at the Federal University of Pará. The objective is to understand the process of poetic use of embedded systems with a focus on the Arduino platform, analyzing and comparing some existing productions that use this technology to three interactive installations done by the author: an antique green analog telephone altered to simulate bird's calls, a tap that emits people speech about water through a headphone connected to it on the gallery wall and an interactive umbrella equipped with speakers connected to an electronic carpet capable of telling where the user is standing holding the umbrella to play specific city's soundscape. All three interactive sonic installations work with previously recorded sounds of different areas of the city of Belem, located at the entrance of the Amazon River, to reflect about the relationship between man, technology and nature. The birds singing on the phone were species usually found on the city of Belem in the past but that are harder or impossible to find it today on the same area, the people interviewed for the tap installation were residents of Belém who live in different neighborhoods and for the umbrella installation were recorded three different soundscapes of the city for: the urban, the fluvial and the forest soundscape. The installations where financed by the Cultural Foundation of the State of Pará and were displayed in a exhibition called "Interurbano" (2018) for public interaction at the Kamara Kó Gallery. The paper addresses the main questions that started the creative process and the relation of the works with the city of Belém in the Amazon Rain Forest, the creative process of the author and collaboration with technicians using microcontrollers in artistic installations as well as the concepts of interactivity, interface, imagery, and augmented space within aesthetic proposals focused on art and technology. The creation of the work will be covered from the author's first ideas, drawings, schematics, prototyping, wiring and coding with Arduino to the installation montage and public interaction at the gallery. All the process was recorded in video as well as photographs showing the nuances of the work in progress. Several artistic proposals are presented with poetic use of microcontrollers to alter objects and develop installations with interactive or responsive interfaces to the environment. All the code done in the project is shared with the public along with schematics of the works. The paper also approaches the works of other artists such as Robyn Farah, Gilberto Prado, Nils Volker and Daniel Rosin comparing different uses of microcontrollers in art installations. 